\section{Mitochondriale Enzephalomyopathien}

\author{
Marcus Deschauer, \\ Klinik und Poliklinik für Neurologie der Martin-Luther-Universität Halle-Wittenberg, Halle/Saale
}

psychoneuro 2003; 29 (3): 108-112

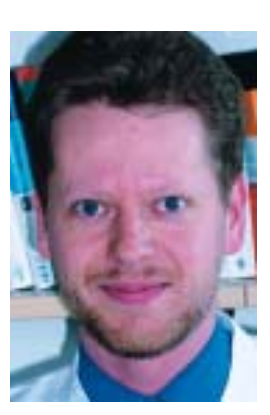

Marcus Deschauer
Mitochondriale Enzephalomyopathien beruhen auf Störungen in der Atmungskette und zeigen sich klinisch häufig als Multisystemerkrankung mit sehr unterschiedlichen Symptomen, wobei insbesondere die Muskulatur und das Nervensystem betroffen sind (z.B. externe Ophthalmoplegie, Extremitätenschwäche, epileptische Anfälle). Erkrankungsbeginn ist meist das Kindesund frühe Erwachsenenalter. Molekulargenetisch können den mitochondrialen Enzephalomyopathien sowohl Defekte der mitochondrialen DNA als auch Mutationen in der Kern-DNA zugrunde liegen. Dies bedingt verschiedene Erbgänge (maternal, autosomal dominant oder rezessiv), aber auch sporadisches Auftreten kommt vor. Neben der molekularen Diagnostik sind ein erhöhter Laktatwert im Blut und Liquor sowie eine Muskelbiopsie, in der sich histologisch (Ragged-Red-Fasern und COX-negative-Fasern) sowie biochemisch Mitochondrienstörungen nachweisen lassen, diagnostisch hilfreich. Mitochondriale Enzephalomyopathien lassen sich bislang nur symptomatisch behandeln.
$\mathrm{M}$ itochondriale Enzephalomyopathien beruhen auf Störungen in der mitochondrialen Atmungskette. Aufgabe der Atmungskette ist die Synthese des energiereichen Adenosintriphosphats (ATP) in der oxidativen Phosphorylierung. Man bezeichnet die mitochondrialen Enzephalomyopathien daher auch als OXPHOS-Defekte. Die Atmungskette besteht aus fünf Enzymkomplexen (Komplex I$\mathrm{V})$, die in die Innenmembran der Mitochondrien integriert sind. Die Mitochondrien gelten als die „Kraftwerke“ der Zellen. Alle wichtigen Reaktionen der Zelle (Muskelkontraktion, Aufbau von Ionengradienten, Synthese von Nukleinsäuren, Proteinen, Lipiden und Kohlenhydraten) werden durch ATP getrieben. Mitochondrienerkrankungen, die andere Stoffwechselwege in den Mitochondrien betreffen, z.B. die Beta-Oxidation der Fettsäuren, gehören im engeren Sinne nicht zu den mitochondrialen Enzephalomyopathien.

\section{Genetische Grundlagen}

Mitochondrien sind die einzigen subzellulären Organellen, die eine eigene DNA besitzen. Das mitochondriale Genom besteht aus einer ringförmigen, doppelsträngigen DNA von 16,5 kB Länge, die in mehreren tausend Kopien in jeder Zelle vorliegt (Abb. 1). Dreizehn Untereinheiten der Atmungskette, zwei rRNA und 22 tRNA werden durch die mitochondriale DNA (mtDNA) kodiert. Der Großteil der Untereinheiten der Atmungskette wird jedoch durch die nukleäre DNA kodiert und in die
Mitochondrien importiert. Deshalb sind unterschiedliche Vererbungsmodi möglich. Je nach zugrundeliegender Mutation wird entweder ein mitochondrialer, d.h. maternaler Erbgang, oder ein autosomaler Erbgang beobachtet.

Der maternale Vererbungsmodus stellt eine spezifische Eigenschaft der mitochondrialen Genetik dar. Die Oozyte enthält einige hunderttausend mtDNA-Kopien, die Spermien hingegen nur etwa hundert, mit der Konsequenz, dass der geringe Anteil paternaler mtDNA kaum einen Einfluss auf den Genotyp hat. So vererben Mütter ihre mtDNA allen ihren Nachkommen, wogegen nur die Töchter die mtDNA an die nächste Generation weitergeben.

Treten in der mtDNA Mutationen auf, so sind diese innerhalb einer Zelle nur bei einem Teil der mtDNAKopien zu finden. Dieser Zustand mit einem Gemisch von mutierter DNA und Wildtyp-DNA wird als Heteroplasmie bezeichnet. Der Anteil mutierter DNA kann von Gewebe zu Gewebe unterschiedlich sein und ist meist in betroffenen Organen am höchsten. Man nimmt an, dass ein bestimmter Schwellenwert an mutierter DNA erreicht werden muss, damit Mutationen manifest werden. Daher kann man auch bei klinisch 
nicht betroffenen Familienangehörigen eine Mutation in geringerem Ausmaß finden. Allerdings korreliert der Heteroplasmiegrad nicht eng mit der klinischen Ausprägung. Bisher sind über 100 Mutationen in der mtDNA nachgewiesen worden. Die Genotyp-Phänotyp-Korrelation ist häufig gering. Einerseits kann eine Mutation verschiedene Syndrome bedingen, andererseits ein Syndrom durch verschiedene Mutationen hervorgerufen werden. Auch in nukleären Genen, die für Untereinheiten der Atmungskette kodieren, wurden (insbesondere bei Manifestation im Kindesalter) Mutationen gefunden (1, 9). Außerdem gibt es Patienten mit autosomalem Erbgang und multiplen Deletionen oder Mangel an mtDNA, bei denen ein Kommunikationsdefekt zwischen nukleärem und mitochondrialem Genom besteht. Es finden sich Mutationen in nuklearen Genen, die für die Replikation der mtDNA mitverantwortlich sind (7).

\section{Symptomatik}

Es besteht eine große Variabilität der klinischen Symptomatik, da verschiedene Organe betroffen sein können, wie in Abbildung 2 dargestellt ist. Bevorzugt sind Nervensystem und Muskulatur betroffen, daher auch die Bezeichnung mitochondriale Enzephalomyopathien. Häufig besteht darüber hinaus eine Multisystemerkrankung. Die gelegentlich verwendete Bezeichnung „mitochondriale Zytopathie“, die den Multisystemcharakter zum Ausdruck bringen soll, erscheint jedoch nicht gerechtfertigt, da letztlich alle genetischen Erkrankungen in jeder Zelle eine Mutation aufweisen und viele monogenetisch bedingte Erkrankungen mehrere Organsysteme betreffen können. Erkrankungsbeginn ist meist das Kindes- und frühe Erwachsenenalter, es gibt jedoch auch Fälle mit Erstmanifestation im höheren Lebensalter (6). Charakteristisch für eine mitochondriale Enzephalomyopathie ist insbesondere eine chronisch progressive externe Ophthalmoplegie (CPEO), die durch Augenbewegungsstörung und Ptosis (Abb. 3) gekennzeichnet ist. Vielfach haben Patienten mit CPEO auch eine
Multisystembeteiligung (CPEO plus) (14). Darüber hinaus gibt es einige charakteristische Symptomkombinationen, die als Syndrome beschrieben wurden. Dazu gehört z.B. das MELAS-Syndrom (Mitochondriale Myopathie, Enzephalopathie, Laktatazidose und schlaganfallähnliche Episoden) oder das MERRF-Syndrom (Myoklonus Epilepsie mit Ragged-red-Fasern). Bei CPEO mit gastrointestinaler Symptomatik spricht man vom MNGIE-Syndrom (mitochondriale neuro-gastro-intestinale Enzephalomyopathie). Unter dem NARP-Syndrom versteht man die Kombination aus Neuropathie, Ataxie und Retinitis pigmentosa. Es gibt jedoch auch Manifestationsformen, die im Wesentlichen auf ein Organ beschränkt sind, wie die Lebersche hereditäre Optikusneuropathie (LHON), die bevorzugt

\section{Abb.1 Mitochondriale DNA mit Genverteilung und Mutationen}
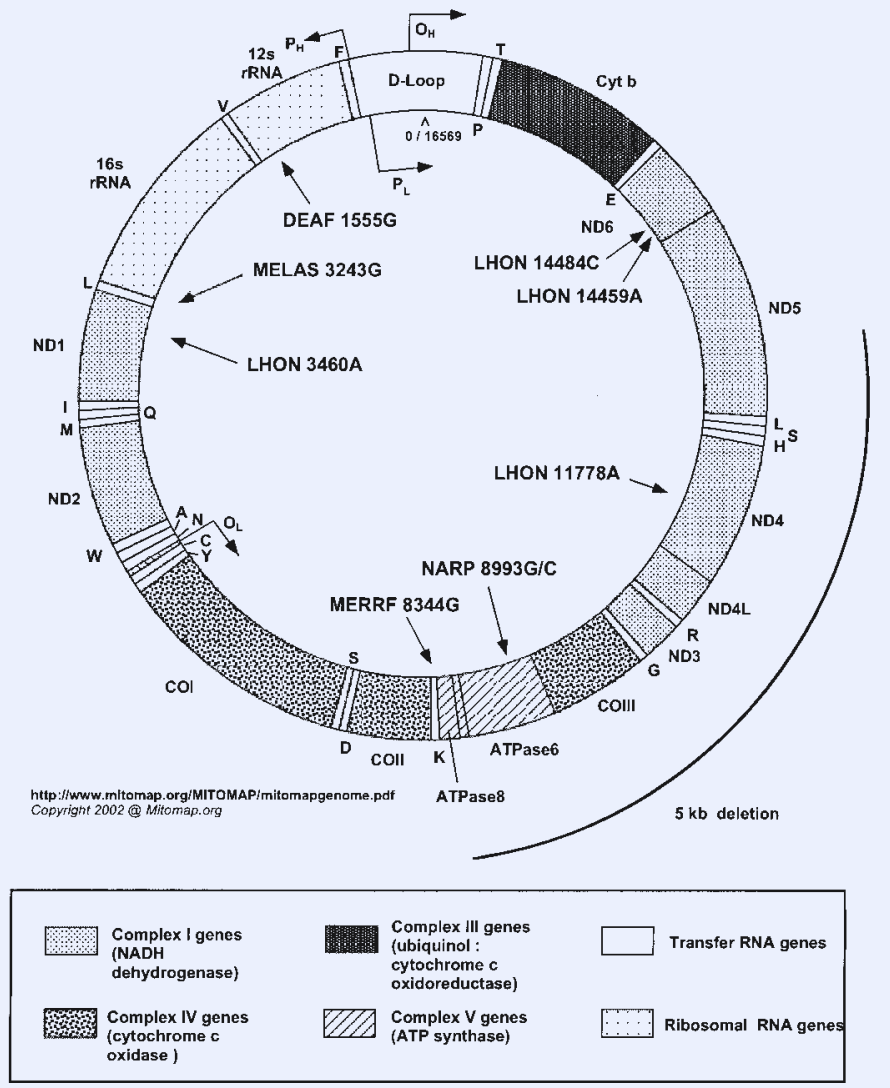

Die wichtigsten Punktmutationen mitochondrialer Enzephalomyopathien sind mit der Nukleotidposititon vermerkt und die Lokalisation der häufigsten mitochondrialen Deletion („common deletion“) ist dargestellt. 


\section{Abb. 2 Multiorganbeteiligung mitochondrialer Enzephalomyopathien}

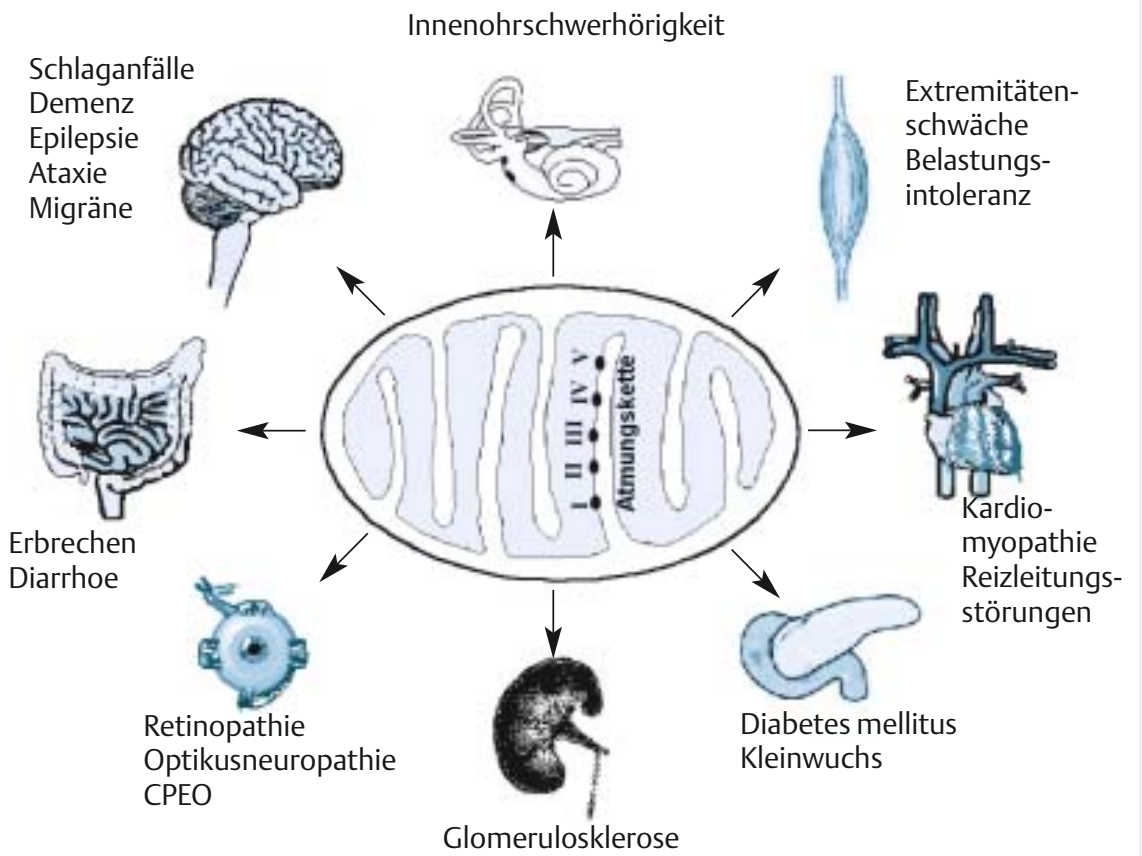

höhter Laktatwert im Blut oder Liquor (und auch erhöhter Laktat/Pyruvat-Quotient) ist demnach ein wichtiger Indikator für das Vorliegen einer mitochondrialen Enzephalomyopathie. Häufig findet sich in Ruhe aber ein normaler Laktatwert im Blut. Unter leichter Belastung im Fahrrad-Belastungs-Test (15 Minuten bei $30 \mathrm{~W}$ ) kommt es jedoch häufig bei Patienten zu einem pathologischen Laktat-Anstieg, der bei Gesunden ausbleibt (13).

\section{Molekulargenetische}

Untersuchungen

In der mitochondrialen DNA finden sich Punktmutationen und auch große Deletionen, die bis zu einige tausend Basen umfassen können. Punktmutationen der mtDNA werden maternal vererbt und lassen sich in DNA aus EDTA-Blut sicher nachweisen. Als häufigste Punktmutation gilt die Mutation A3243G, die sich bei rund $80 \%$ der Patienten mit MELAS-Syndrom findet, die jedoch auch verschiedene andere Phänotypen aufweisen (z.B. CPEO plus, Dia-

\section{Tab. 1 Wichtige Syndrome bei mitochondrialen Enzephalomyopathien}

\begin{tabular}{|c|c|c|c|}
\hline Syndrom & Symptomatik & Erbgang & Gendefekt \\
\hline CPEO plus & $\begin{array}{l}\text { Augenbewegungsstörung mit Ptosis, } \\
\text { Extremitätenschwäche, Retinopathie, } \\
\text { Reizleitungsstörungen des Herzens, } \\
\text { Psychosyndrom/Demenz, Polyneuropathie, } \\
\text { Kleinwuchs, Diabetes mellitus, Ataxie, Hypakusis }\end{array}$ & $\begin{array}{l}\text { Sporadisch } \\
\text { Autosomal rezessiv } \\
\text { oder dominant } \\
\text { Maternal }\end{array}$ & $\begin{array}{l}\text { Singuläre Deletion der mtDNA } \\
\text { Multiple Deletionen der mtDNA } \\
\text { Mutation A3243G der mtDNA }\end{array}$ \\
\hline MELAS & $\begin{array}{l}\text { Schlaganfallähnliche Episoden (insbesondere mit } \\
\text { Hemianopsie), Hypakusis, Übelkeit/Erbrechen, } \\
\text { Kopfschmerzen, epileptische Anfälle, Psycho- } \\
\text { syndrom/Demenz, Diabetes mellitus, Kleinwuchs }\end{array}$ & Maternal & Mutation A3243G der mtDNA \\
\hline MERRF & $\begin{array}{l}\text { Myokloni, generalisierte Krampfanfälle, Ataxie, } \\
\text { Hypakusis, Extremitätenschwäche, Psychosyndrom/ } \\
\text { Demenz, Optikusatrophie, Polyneuropathie }\end{array}$ & Maternal & Mutation A8344G der mtDNA \\
\hline LHON & $\begin{array}{l}\text { Rasch fortschreitende Visusminderung mit } \\
\text { zentralem Skotom, meist einseitig beginnend und } \\
\text { nach einigen Wochen auch das andere Auge } \\
\text { betreffend, initial Papillenödem, später Atrophie }\end{array}$ & Maternal & $\begin{array}{l}\text { Mutation G11778A der mtDNA } \\
\text { Mutation G3460A der mtDNA } \\
\text { Mutation T14484C der mtDNA }\end{array}$ \\
\hline Leigh & $\begin{array}{l}\text { Im frühen Kindesalter Muskelhypotonie, } \\
\text { psychomotorische Retardierung, Ophthalmoplegie, } \\
\text { Ptosis, Nystagmus, Dystonie, Saug- bzw. Schluck- } \\
\text { schwierigkeiten, zerebrale Krampfanfälle }\end{array}$ & $\begin{array}{l}\text { Meist autosomal } \\
\text { rezessiv } \\
\text { Seltener maternal }\end{array}$ & $\begin{array}{l}\text { Mutationen im SURF1-Gen } \\
\text { Mutationen im SCO2-Gen } \\
\text { Mutation T8993G/C der mtDNA }\end{array}$ \\
\hline NARP & $\begin{array}{l}\text { Sensible Neuropathie, Ataxie, Retinitis pigmentosa, } \\
\text { epileptische Anfälle }\end{array}$ & Maternal & Mutation T8993G/C der mtDNA \\
\hline MNGIE & $\begin{array}{l}\text { Augenbewegungsstörung mit Ptosis, } \\
\text { Darmmotilitätsstörung, Diarrhoe, Pseudo- } \\
\text { obstruktion, Übelkeit/Erbrechen, Kachexie, } \\
\text { Polyneuropathie, Leukenzephalopathie }\end{array}$ & Autosomal rezessiv & $\begin{array}{l}\text { Multiple Deletionen der mtDNA } \\
\text { Depletion (Mangel) der mtDNA }\end{array}$ \\
\hline
\end{tabular}




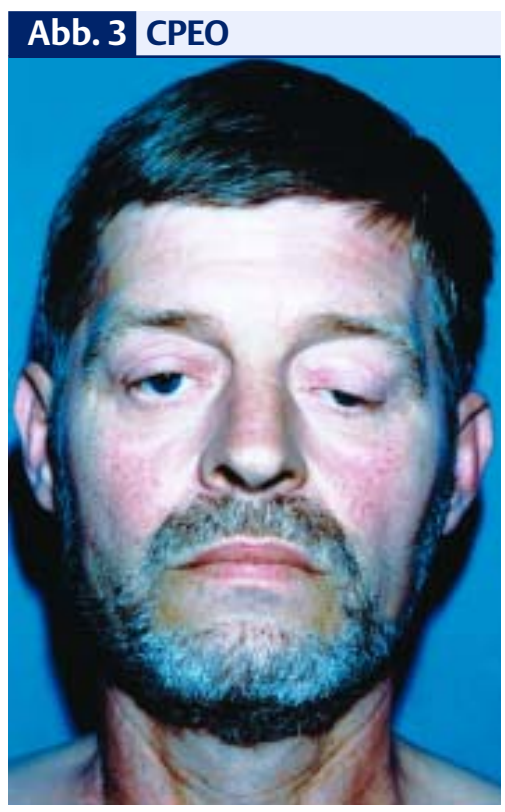

50-jähriger Patient mit der Punktmutation A3243G der mtDNA.

betes und Hörstörung, schmerzhafte Muskelsteife) (3). Besonders häufig haben Patienten mit dieser Mutation eine Hörstörung (5).

Bei Patienten mit MERRF-Syndrom findet man in rund $80 \%$ die Mutation A8344G. Etwa die Hälfte der Patienten mit LHON tragen die Mutation G11778A, seltener die Mutation G3460A und T14484C (1). Die große Mehrzahl der Patienten mit CPEO weisen Deletionen der mtDNA auf. Man muss dabei zwischen singulären Deletionen, die in der Regel sporadisch auftreten, und multiplen Deletionen unterscheiden, die autosomal dominant oder rezessiv vererbt werden (4). Der autosomale Erbgang bei multiplen Deletionen lässt auf einen primär nukleären Gendefekt schließen, der sekundär durch eine Replikationsstörung der mtDNA zu den multiplen Deletionen führt. Mutationen wurden in folgenden nuklearen Genen gefunden: Twinkle, Polymerase Gamma 1, Adenosin-Nukleotid-Translokator 1 und Thymidin-Phosphorylase $(7,8,12)$.

Beim Leigh-Syndrom kann man Mutationen an Position 8993 der mtDNA finden. Bei rezessiven Fällen mit Komplex IV-Mangel lassen sich Mutationen im nukleären Gen SURF1 nachweisen, das für die Kombination der Komplex IV-Untereinheiten wichtig ist. Kinder mit Leigh-ähnli- cher Symptomatik und Kardiomyopathie können Mutationen im SCO2-Gen aufweisen, das ebenfalls für die Kombination der Komplex IVUntereinheiten mitverantwortlich ist (1).

\section{Muskelbiopsie}

Insbesondere bei Patienten mit MELAS, MERRF und CPEO (nicht jedoch bei LHON) finden sich in der Gomori-Trichrom-Färbung Raggedred-Fasern. Hierbei handelt es sich um Muskelfasern, die fuchsinrote, überwiegend subsarkolemmal gelegene Mitochondrien-Agglomerationen aufweisen, wobei das intermyofibrilläre Netzwerk vielfach fragmentiert bzw. zerrissen erscheint. Häufig findet man bei Patienten mit mitochondrialen Enzephalomyopathien eine Beeinträchtigung des Komplex IV der Atmungskette (Cytochrom-C-Oxidase $=$ COX), die sich histochemisch durch COX-negative Fasern nachweisen lässt (Abb. 4). Auch biochemisch kann man im Muskelhomogenat erniedrigte Aktivitäten der Atmungsketten-Komplexe messen $(9,10)$.

\section{Zerebrale Bildgebung}

Beim MELAS-Syndrom sieht man im CT häufig Hypodensitäten, die besonders okzipital und parietal lokalisiert sind. Die Läsionen sind gewöhnlich bilateral, symmetrisch oder asymmetrisch. Eine Erweiterung der äußeren und inneren Liquorräume und Ausziehung der Okzipitalhörner der Seitenventrikel sowie Basalganglienverkalkungen sind häufig. In der Kernspintomographie zeigen die Läsionen gewöhnlich ein verlängertes T2-Signal. Sie lassen sich keinem einzelnen Gefäßterritorium oder der Grenzzone zweier Gefäßterritorien zuordnen. Beim M. Leigh findet man im CT symmetrische Hypodensitäten vorwiegend in den Stammganglien bzw. T1-Hypointensitäten sowie T2-Hyperintensitäten im $\operatorname{MRT}(9,11)$.

\section{- Therapieansätze}

Eine kurative Therapie mitochondrialer Enzephalomyopathien existiert nicht. Es wurden versucht durch die Supplementation von Coenzymen und Vitaminen, die für die Atmungskette wichtig sind, die Erkrankungen positiv zu beeinflussen, ohne dass bisherige Studien eine eindeutige Wirksamkeit zeigen konnten. Dabei kamen insbesondere Coenzym Q, Creatin, Carnitin, Vitamin B1 und B2, Vitamin C und Vitamin K zum Einsatz. Da keine wesentlichen Nebenwirkungen zu befürchten sind und keine anderen

\section{Tab. 2 Zusatzuntersuchungen zur klinisch-neurologischen Untersuchung bei Verdacht auf eine mitochondriale Enzephalomyopathie}

\begin{tabular}{ll} 
Untersuchung & Fragestellung \\
\hline Labor & $\begin{array}{l}\text { Laktaterhöhung Blut/Liquor } \\
\text { Fahrradbelastungstest } \\
\text { CK-Erhöhung } \\
\text { Diabetes }\end{array}$ \\
\hline Elektrophysiologie & $\begin{array}{l}\text { Neuropathie oder Myopathie im EMG/NLG } \\
\text { Epilepsietypische Potenziale im EEG }\end{array}$ \\
\hline Kardiologische Untersuchung & $\begin{array}{l}\text { Reizleitungsstörungen im EKG } \\
\text { Kardiomyopathie im Herzecho/Rö-Thorax }\end{array}$ \\
\hline Zerebrale Bildgebung & $\begin{array}{l}\text { "MELAS-Läsionen“ } \\
\text { Stammganglienläsionen }\end{array}$ \\
\hline HNO-Ärztliche Untersuchung & Stammganglienverkalkungen \\
\hline Augenärztliche Untersuchung & Retinopathie, Optikusatrophie \\
\hline Muskelbiopsie & $\begin{array}{l}\text { Ragged-Red-Fasern } \\
\text { COX-negative Fasern }\end{array}$ \\
& $\begin{array}{l}\text { Erniedrigung von Komplexen der } \\
\text { Atmungskette }\end{array}$ \\
\hline Molekulargenetik & Nachweis von Mutationen \\
\hline
\end{tabular}




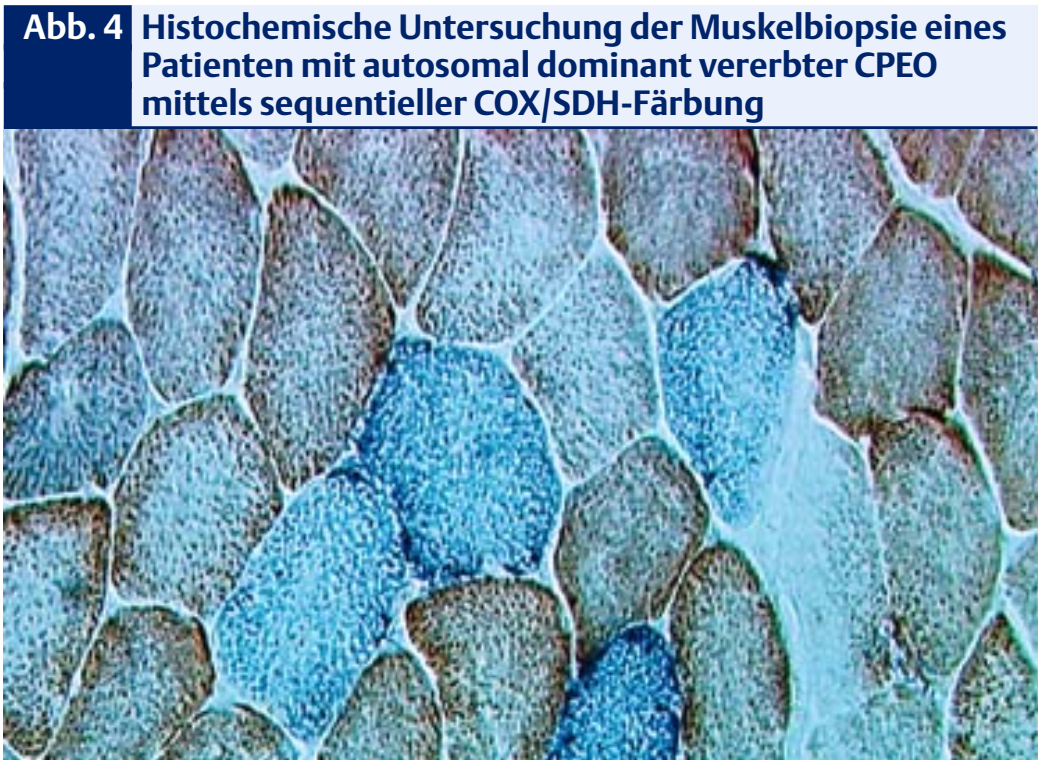

In Fasern mit normaler COX-Aktivität zeigt sich eine braune Anfärbung, während sich COX-negative Fasern blau färben. Das blaue Reaktionsprodukt der SDH entsteht nur in den COX-negativen Fasern, da die COX-positiven Fasern bereits mit dem braunen Endprodukt der COX-Reaktion gesättigt sind.

medikamentösen Alternativen zur Verfügung stehen, erscheint im Einzelfall eine probatorische Gabe gerechtfertigt.

Eine lebensrettende Maßnahme ist dagegen jedoch die rechtzeitige Implantation eines Schrittmachers bei Reizleitungsstörungen des Herzens, die häufig bei CPEO, aber auch bei anderen Syndromen, auftreten. Eine Ptosis kann operativ durch Lidraffung behandelt werden. Bei der antiepileptischen Therapie sollte aus theoretischen Überlegungen auf Valproat verzichtet werden. Moderates körperliches Training kann die bei mitochondrialen Enzephalomyopathien häufig vorliegende Belastungsintoleranz vermindern. Es bleibt zu hoffen, dass zukünftig gentherapeutische Maßnahmen eine wirksame Heilmethode der mitochondrialen Enzephalomyopathien bieten können (2).

Mitochondrial Enzephalomyopathies are caused by defects of the respiratory chain. Frequently they present with a multisystemic involvement and predominantly muscle and nervous system are affected (e.g. external ophthalmoplegia, limb weakness, seizures). Typically, onset is in childhood or early adulthood. Underlaying molecular defects are localized both in mitochondrial DNA and nuclear DNA. Consequently, there are different modes of inheritance (maternal and autosomal recessive or dominant) but sporadic occurrence is possible, too. Besides molecular testing an elevated lactate in blood or cerebral spinal fluid has diagnostic value. Histological changes of mitochondria (ragged-redfibers and COX-negative fibers) and decreased activities of respiratory chain are found in a muscle biopsy specimens. At the moment only smptomatic therapy is available.

\section{Literatur:}

1. Chinnery PF, Turnbull DM. Mitochondrial DNA mutations in the pathogenesis of human disease. Mol Med Today 2000; 6 : 425-432

2. Chinnery PF, Turnbull DM: Epidemiology and treatment of mitochondrial disorders. Am J Med Genet (Semin Med Genet) 2001; 106: 94-101

3. Deschauer M, Wieser T, Neudecker $S$, Lindner A, Zierz S. Mitochondrial $3243 \mathrm{~A} \rightarrow \mathrm{C}$ mutation (MELAS mutation) associated with painful muscle stiffness. Neuromuscular Disorders 1999; 9: 305-307

4. Deschauer M, Müller T, Dreha S, Zierz S. Familiäre mitochondriale chronisch progressive externe Ophthalmoplegie - Fünf Familien mit unterschiedlicher Genetik. Nervenarzt 2001; 72: 122-129 Mattler W, Kornhuber M, Zierz S. Hearing impairment is common in various phenoty-
5. Deschauer M, Müller T, Wieser T, Schulte- pes of the mitochondrial DNA A3243G mutation. Archives of Neurology 2001; 58: 1885-1888

6. Deschauer M, Bamberg C, Claus D, Zierz S, Turnbull DM, Taylor RW. Late-onset encephalopathy associated with a C11777A mutation of mitochondrial DNA. Neurology (im Druck)

7. Deschauer M, Zierz S. Defekte der intergenomischen Kommunikation: Mutationen der Kern-DNA und multiple Deletionen der mitochondrialen DNA bei chronisch progressiver externer Ophthalmoplegie. Aktuelle Neurologie (im Druck)

8. Deschauer M, Kiefer R, Blakely EL, Zierz S, Turnbull DM, Taylor RW. A novel Twinkle gene mutation in autosomal dominant progressive external ophthalmoplegia. Submitted

9. DiMauro S, Andreu AL, Musumeci O, Bonilla E. Diseases of oxidative phosphorylation due to mtDNA mutations. Semin Neurol 2001; 21:251-60

10. Gellerich FN, Deschauer M, Müller T, Chen Y, Opalka JR, Zierz S. Mitochondrial respiratory rates and activities of respiratory chain complexes correlate linearly with heteroplasmy of deleted mtDNA without threshold and independently of deletion size. Biochim Biophys Acta 2002 1556(1): 41-52

11. Lindner A, Hofmann E, Naumann M, Becker G, Reichmann H. Clinical, morphological, biochemical, and neuroradiological features of mitochondrial encephalomyopathies. Presentation of 19 patients. Mol Cell Biochem 1997; 174: 297-303

12. Suomalainen A, Kaukonen J. Diseases caused by nuclear genes affecting mtDNA stability. Am J Med Genet (Sem Med Genet) 2001; 106: 53-61

13. Zierz S, Meeßen S, Jerusalem F. Laktatund Pyruvatblutspiegel in der Diagnostik mitochondrialer Myopathien. Nervenarzt 1989; 60: 545-548

14. Zierz S, v. Wersebe O, Gerbitz KD, Jerusalem F. Ophthalmoplegia plus: klinische Variabilität, biochemische Defekte der mitochondrialen Atmungskette und Deletionen des mitochondrialen Genoms. Nervenarzt 1990; 61: 332-339

\section{Korrespondenzadresse:}

Dr. med. Marcus Deschauer

Klinik und Poliklinik für Neurologie

Martin-Luther-Universität Halle-Wittenberg Ernst-Grube-Str. 40 06097 Halle/Saale

e-mail: marcus.deschauer@medizin.unihalle.de 